Ann. Funct. Anal. 6 (2015), no. 4, 215-225

http://doi.org/10.15352/afa/06-4-215

ISSN: 2008-8752 (electronic)

http://projecteuclid.org/afa

\title{
ADJOINTS OF GENERALIZED COMPOSITION OPERATORS WITH RATIONAL SYMBOL
}

\author{
ALIAKBAR SALARYAN* AND HAMID VAEZI \\ Communicated by K. Zhu
}

\begin{abstract}
Given $\varphi: \mathbb{U} \rightarrow \mathbb{U}$, an analytic self-map of the open unit disc in complex plane, the composition operator $C_{\varphi}$ is defined by $C_{\varphi} f=f \circ \varphi$ for $f$ belonging to some Hilbert space of analytic functions on $\mathbb{U}$. In the present paper, we introduce a generalization of the composition operators and reproducing kernel functions on the weighted Hardy spaces. We also obtain the adjoints of generalized composition operators with rational symbol acting on the Hardy, Bergman and Dirichlet spaces.
\end{abstract}

\section{Introduction}

Let $\mathbb{U}$ denote the open unit disc of the complex plane. For each sequence $\beta=$ $\left\{\beta_{n}\right\}$ of positive numbers, the weighted Hardy space $H^{2}(\beta)$ consists of analytic functions $f(z)=\sum_{n=0}^{\infty} a_{n} z^{n}$ on $\mathbb{U}$ for which the norm

$$
\|f\|_{\beta}=\left(\sum_{n=0}^{\infty}\left|a_{n}\right|^{2} \beta_{n}^{2}\right)^{\frac{1}{2}}
$$

is finite. Notice that the above norm is induced by the following inner product

$$
\left\langle\sum_{n=0}^{\infty} a_{n} z^{n}, \sum_{n=0}^{\infty} b_{n} z^{n}\right\rangle_{\beta}=\sum_{n=0}^{\infty} a_{n} \overline{b_{n}} \beta_{n}^{2},
$$

and that the monomials $z^{n}$ form a complete orthogonal system for $H^{2}(\beta)$. Consequently, the polynomials are dense in $H^{2}(\beta)$ (see [4, Section 2.1]). Every weighted

Date: Received: Mar. 13, 2015; Accepted: Jun. 7, 2015.

* Corresponding author.

2010 Mathematics Subject Classification. Primary 47B33; Secondary 47B38, 47A05.

Key words and phrases. Generalized composition operator, adjoint, reproducing kernel, weighted Hardy space. 
Hardy space $H^{2}(\beta)$ contains a family of the reproducing kernels $\left\{K_{w}: w \in \mathbb{U}\right\}$; that is, $\left\langle f, K_{w}\right\rangle_{\beta}=f(w)$ for every $f \in H^{2}(\beta)$. Indeed, $K_{w}(z)=\sum_{n=0}^{\infty} \frac{1}{\beta_{n}^{2}} \bar{w}^{n} z^{n}$. Observe that particular instances of the sequence $\beta=\left\{\beta_{n}\right\}$ yield well-known Hilbert spaces of analytic functions. In particular, $\beta_{n}=1$ corresponds to the Hardy space $H^{2}(\mathbb{U})$. If $\beta_{0}=1$ and $\beta_{n}=n^{1 / 2}$ for $n \geq 1$, the resulting space is the classical Dirichlet space $\mathcal{D}$ and if $\beta_{n}=(n+1)^{-1 / 2}$, we have the Bergman space $A^{2}(\mathbb{U})$. The reproducing kernel function $K_{w}$ at the point $z \in \mathbb{U}$ for the Hardy, Bergman and Dirichlet spaces is given by

$$
\frac{1}{1-\bar{w} z}, \quad \frac{1}{(1-\bar{w} z)^{2}}, \quad 1+\log \frac{1}{1-\bar{w} z}
$$

respectively.

The inner product of two functions $f$ and $g$ in any of these spaces may also be computed by integration. For the Hardy space $H^{2}(\mathbb{U})$,

$$
\langle f, g\rangle_{H^{2}(\mathbb{U})}=\int_{0}^{2 \pi} f\left(e^{i \theta}\right) \overline{g\left(e^{i \theta}\right)} \frac{d \theta}{2 \pi}
$$

where $f$ and $g$ are defined a.e. on $\partial \mathbb{U}$ via radial limits (see [5]). In the case of the Bergman space,

$$
\langle f, g\rangle_{A^{2}(\mathbb{U})}=\int_{\mathbb{U}} f(z) \overline{g(z)} d A(z),
$$

where $d A$ is normalized area measure on $\mathbb{U}$. For the Dirichlet space, the inner product is given by

$$
\langle f, g\rangle_{\mathcal{D}}=f(0) \overline{g(0)}+\int_{\mathbb{U}} f^{\prime}(z) \overline{g^{\prime}(z)} d A(z) .
$$

If $u$ is analytic on the open unit disc $\mathbb{U}$ and $\varphi$ analytic self-map of the unit disc, the weighted composition operator on $H^{2}(\beta)$ with symbols $u$ and $\varphi$ is the operator $\left(W_{u, \varphi} f\right)(z)=u(z) f(\varphi(z))$ for $f$ in $H^{2}(\beta)$. When $u(z) \equiv 1$ we call the operator a composition operator and denote it by $C_{\varphi}$. The multiplication operator $M_{u}$ corresponds to the case $\varphi(z)=z$ and is given by $M_{u} f(z)=u(z) f(z)$. For general information in this context, one can see the excellent monographs [4, 14].

In recent years the concept of composition and weighted composition operators has been generalized in the literature. The generalized weighted composition operator $D_{\varphi, u}^{n}$, which was introduced by Zhu [16] (see also [15]), is defined

$$
\left(D_{\varphi, u}^{n} f\right)(z)=u(z) f^{(n)}(\varphi(z))
$$

where $f^{(n)}$ denotes the $n$th derivative of $f$ for $n \geq 0$. Another generalization of composition operators may be defined via anti-derivatives:

$$
C_{\varphi}^{[n]} f(z)=f^{[n]}(\varphi(z))
$$

where $f^{[n]}$ denotes the $n$th anti-derivative of $f$ with $f^{[1]}(0)=f^{[2]}(0)=\cdots=$ $f^{[n]}(0)=0$. One reason to study this type of operators is that they appear in the adjoint formula for rationally induced composition operators on the Bergman space (see [7, Theorem 3.5, Corollary 3.6]). 
One of the most fundamental questions relating to composition and generalized composition operators is how to obtain a reasonable representation for their adjoints. The problem of computing the adjoint of a composition operator induced by a linear fractional symbol on the Hardy space was solved by Cowen [2]. Hurst [9] used an analogous argument to obtain the solution in the weighted Bergman space $A_{\alpha}^{2}(\mathbb{U})$. In both of these cases, the adjoint consists of products of a composition operator, a Toeplitz operator and the adjoint of another Toeplitz operator. In 2003, Gallardo-Gutiérrez and Montes-Rodríguez [6] computed the adjoint of a composition operator acting on the Dirichlet space by a different method from those used by Cowen and Hurst. Hammond, Moorhouse and Robbins [8] solved the case for rationally induced composition operators on the Hardy space $H^{2}(\mathbb{U})$. Bourdon and Shapiro [1] reproduced the Hammond-Moorhouse-Robbins formula in a straightforward algebraic fashion. The authors in [7] gave the adjoint formula for rationally induced composition operators on the Dirichlet and Bergman spaces. For more details we refer interested reader to [3, 12, 13].

In Section 3 of this paper we construct generalized reproducing kernel functions on the weighted Hardy spaces. In Section 4, after a summary discussion of boundedness, we investigate the adjoint of a generalized composition operator

$C_{\varphi}^{[n]}$. Then for a rational self-map $\varphi$ of the open unit disc, we obtain the adjoint of $C_{\varphi}^{[1]}$ on the Hardy, Bergman and Dirichlet spaces.

\section{Preliminary results}

Let $H^{2}(\gamma)$ and $H^{2}(\beta)$ denote the weighted Hardy spaces with weight sequences $\left\{\gamma_{n}\right\}$ and $\left\{\beta_{n}\right\}$, respectively . Then $H^{2}(\gamma) \cap H^{2}(\beta)$ contains all polynomials and hence is not empty. The following theorem and its corollary (see [7]) are necessary for our work .

Theorem 2.1. Let $T_{0}$ and $T_{1}$ be bounded operators on $H^{2}(\gamma)$ and $H^{2}(\beta)$ respectively, such that $T_{0} p=T_{1} p$ for any polynomial $p$, and define $T: H^{2}(\beta) \rightarrow H^{2}(\gamma)$ by $T\left(\sum_{n=0}^{\infty} a_{n} z^{n}\right)=\sum_{n=0}^{\infty} a_{n} \frac{\beta_{n}}{\gamma_{n}} z^{n}$. The operator $T$ is invertible, and

(i) $T^{-1} T_{0}^{*} T p=T T_{1}^{*} T^{-1} p$ for any polynomial $p$.

(ii) If $H^{2}(\gamma) \subset H^{2}(\beta)$ with continuous inclusion, then $T^{-1} T_{0}^{*} T g=T T_{1}^{*} T^{-1} g$ for any $g \in H^{2}(\gamma)$.

Corollary 2.2. Let $H^{2}(\gamma) \subset H^{2}(\beta)$ with continuous inclusion. Then for $T_{0}$, $T_{1}$ and $T$ as in the statement of Theorem 2.1, we have

$$
T_{1}^{*} f=T^{-1}\left(T^{-1}\left(T_{0}^{*}(T(T f))\right)\right), \quad\left(f \in H^{2}(\beta)\right) .
$$

Note that Theorem 2.1 and its corollary cover a wide class of well known operators including (generalized) composition and (generalized) weighted composition operators on weighted Hardy spaces . Hence this theorem may be used to translate results relative to the adjoint problem from one case to another, as we will see in the next section .

Let $\operatorname{Rat}(\mathbb{U})$ denote the collection of all rational functions of one complex variable on $\mathbb{U}$, and let $\varphi$ be a function in $\operatorname{Rat}(\mathbb{U})$ that maps $\mathbb{U}$ into itself . The 
degree of $\varphi$ is the larger of the degrees of its numerator and denominator . Define $\varphi_{e}:=\rho \circ \varphi \circ \rho$, where $\rho$ is defined on extended complex plane $\hat{\mathbb{C}}$ by $\rho(z)=1 / \bar{z}$. If the degree of $\varphi$ is $d$, then for each point $w \in \hat{\mathbb{C}}$ the inverse image $\varphi^{-1}(\{w\})$ has , counting multiplicities, exactly $d$ points. If $\varphi^{-1}(\{w\})$ has $d$ distinct points we will say $w$ is a regular value of $\varphi$. For any rational function, all but finitely many points of $\hat{\mathbb{C}}$ are regular values. The collection of points in $\hat{\mathbb{C}}$ that are regular values of $\varphi$ is denoted by $\operatorname{reg}(\varphi)$. Let $\left\{\sigma_{j}\right\}_{j=1}^{d}$ be $d$ distinct branches of $\varphi_{e}^{-1}$ which are defined on a suitable neighborhood of any regular point of the open unit disc . For more details on above concepts one can see [1] .

Let $\mathcal{H}$ be one of the spaces $H^{2}(\mathbb{U}), A^{2}(\mathbb{U})$ or $\mathcal{D}$ and define $B_{0}: \mathcal{H} \rightarrow \mathcal{H}$ by

$$
\left(B_{0} f\right)(z)=\left\{\begin{array}{cl}
\frac{f(z)-f(0)}{z} & \text { if } z \in \mathbb{U} \backslash\{0\} \\
f^{\prime}(0) & \text { if } z=0
\end{array}\right.
$$

which is just the backward shift operator when $\mathcal{H}=H^{2}(\mathbb{U})$. Let $Q: \mathcal{H} \rightarrow \mathcal{H}$ be defined by $Q f=F$, where $F$ is anti-derivative of $f$ with $F(0)=0$. A rather simple computation involving Maclaurin series shows that both $B_{0}$ and $Q$ are bounded on $\mathcal{H}$. Denote the derivative of $f \in \mathcal{H}$ by $D f=f^{\prime}$. Clearly, $D$ is not a bounded operator on $\mathcal{H}$. We will need the following theorem from [1] .

Theorem 2.3. If $\varphi \in \operatorname{Rat}(\mathbb{U})$, then for each $f \in H^{2}(\mathbb{U})$,

$$
C_{\varphi}^{*} f(z)=\frac{f(0)}{1-\overline{\varphi(0)} z}+z \sum_{j=1}^{d} \sigma_{j}^{\prime}(z)\left(B_{0} f\right)\left(\sigma_{j}(z)\right), \quad\left(z \in \operatorname{reg}\left(\varphi_{e}\right) \cap \mathbb{U}\right) .
$$

Further results from [7] are required in the next section .

Theorem 2.4. Let $\varphi \in \operatorname{Rat}(\mathbb{U})$ and suppose $\mathbb{U} \subseteq \operatorname{reg}\left(\varphi_{e}\right)$. Then for each $f \in \mathcal{D}$ and $z \in \mathbb{U}$,

$$
C_{\varphi}^{*} f(z)=f(0) K_{\varphi(0)}(z)+\sum_{j=1}^{d} C_{\sigma_{j}} f(z)-\sum_{j=1}^{d} C_{\sigma_{j}} f(0) .
$$

Proposition 2.5. Put $H^{2}(\gamma)=H^{2}(\mathbb{U}), H^{2}(\beta)=A^{2}(\mathbb{U})$ and let $T: A^{2}(\mathbb{U}) \longrightarrow$ $H^{2}(\mathbb{U})$ be the operator introduced in Theorem 2.1 for these spaces. Then for any $f \in H^{2}(\mathbb{U})$ with $T^{-1} f \in H^{2}(\mathbb{U})$,

$$
T^{-1}\left(T^{-1} f\right)(z)=(z f(z))^{\prime}=f(z)+z f^{\prime}(z) .
$$

Also for any $f \in A^{2}(\mathbb{U})$,

$$
T(T f)(z)=B_{0} Q f(z)
$$

\section{GenerAlized REPRODUCING KERNEL}

In this section we extend the reproducing kernel property to the higher order anti-derivatives of elements of $H^{2}(\beta)$.

Theorem 3.1. Let $H^{2}(\beta)$ be a weighted Hardy space and let $f^{[n]}$ denote the $n$th anti-derivative of $f$ with $f^{[1]}(0)=f^{[2]}(0)=\cdots=f^{[n]}(0)=0$. For every point $w$ in the open unit disc and every positive integer $n$, the function that takes $f$ 
to $f^{[n]}(w)$ is a bounded linear functional on $H^{2}(\beta)$. Moreover, the corresponding $n$-generalized reproducing kernel function is given by

$$
K_{w, n}(z)=\sum_{m=0}^{\infty} \frac{1}{(m+1) \cdots(m+n) \beta_{m}^{2}} \bar{w}^{m+n} z^{m} .
$$

Proof. For $K_{w, n}$ given in the statement of the theorem we have

$\left\|K_{w, n}\right\|_{\beta}^{2}=\sum_{m=0}^{\infty} \frac{1}{(m+1)^{2} \cdots(m+n)^{2} \beta_{m}^{4}}|w|^{2(m+n)} \beta_{m}^{2}<\sum_{m=0}^{\infty} \frac{1}{\beta_{m}^{2}}|w|^{2 m}=\left\|K_{w}\right\|_{\beta}^{2}$.

Hence $K_{w, n} \in H^{2}(\beta)$ and it is easily seen that for $f \in H^{2}(\beta)$

$$
f^{[n]}(w)=\left\langle f, K_{w, n}\right\rangle_{\beta} .
$$

Now it follows immediately from (3.2) that this functional is linear and bounded on $H^{2}(\beta)$ for $w \in \mathbb{U}$.

It follows from (3.1) that for every weighted Hardy space, $K_{0, n}=0$ for $n \geq 1$. Moreover, we have

$$
K_{w, n}(0)=\frac{1}{n ! \beta_{0}^{2}} \bar{w}^{n}, \quad K_{w, 1}(z)=\sum_{m=0}^{\infty} \frac{1}{(m+1) \beta_{m}^{2}} \bar{w}^{m+1} z^{m} .
$$

Now, we obtain the 1-generalized reproducing kernel $K_{w, 1}$ on the Hardy, Bergman and Dirichlet spaces.

Proposition 3.2. The 1-generalized reproducing kernel function $K_{w, 1}$ on the Hardy space $H^{2}(\mathbb{U})$ is given by

$$
K_{w, 1}(z)=\left\{\begin{array}{cl}
\frac{1}{z} \log \frac{1}{1-\bar{w} z} & \text { if } z \in \mathbb{U} \backslash\{0\} \\
\bar{w} & \text { if } z=0
\end{array}\right.
$$

Proof. It is evident from (3.3) that $K_{w, 1}(0)=\bar{w}$. Again, using (3.3) for $z \neq 0$ we have

$$
\begin{aligned}
K_{w, 1}(z) & =\sum_{m=0}^{\infty} \frac{1}{m+1} \bar{w}^{m+1} z^{m}=\frac{1}{z} \sum_{m=0}^{\infty} \frac{1}{m+1} \bar{w}^{m+1} z^{m+1} \\
& =\frac{1}{z}\left(\sum_{m=0}^{\infty} \bar{w}^{m+1} z^{m}\right)^{[1]}=\frac{\bar{w}}{z}\left(\frac{1}{1-\bar{w} z}\right)^{[1]}=\frac{1}{z} \log \frac{1}{1-\bar{w} z} .
\end{aligned}
$$

Proposition 3.3. The 1-generalized reproducing kernel $K_{w, 1}$ on the Bergman space $A^{2}(\mathbb{U})$ is given by $K_{w, 1}(z)=\frac{\bar{w}}{1-\bar{w} z}$.

Proof. By applying (3.3),

$$
K_{w, 1}(z)=\sum_{m=0}^{\infty} \bar{w}^{m+1} z^{m}=\bar{w} \sum_{m=0}^{\infty}(\bar{w} z)^{m}=\frac{\bar{w}}{1-\bar{w} z} .
$$


Proposition 3.4. The 1-generalized reproducing kernel $K_{w, 1}$ on the Dirichlet space $\mathcal{D}$ is given by

$$
K_{w, 1}(z)=\left\{\begin{array}{cl}
2 \bar{w}+\frac{1-\bar{w} z}{z} \log (1-\bar{w} z) & \text { if } z \in \mathbb{U} \backslash\{0\}, \\
\bar{w} & \text { if } z=0 .
\end{array}\right.
$$

Proof. By using (3.3) for $z \neq 0$,

$$
\begin{aligned}
K_{w, 1}(z) & =\bar{w}+\sum_{m=1}^{\infty} \frac{1}{m(m+1)} \bar{w}^{m+1} z^{m}=\bar{w}+\frac{\bar{w}^{2}}{z} \sum_{m=1}^{\infty}\left((\bar{w} z)^{m-1}\right)^{[2]} \\
& =\bar{w}+\frac{\bar{w}^{2}}{z}\left(\frac{1}{1-\bar{w} z}\right)^{[2]}=2 \bar{w}+\frac{1-\bar{w} z}{z} \log (1-\bar{w} z) .
\end{aligned}
$$

The equality $K_{w, 1}(0)=\bar{w}$ is clear.

\section{Adjoints OF GENERALIZED COMPOSITION OPERATOR WITH RATIONAL SYMBOL}

From now on, we let $Q f=F$ be the anti-derivative of $f$ with $F(0)=0$. Clearly, $Q$ is linear on $H^{2}(\beta)$. In this section we discuss the adjoint of a generalized composition operator $C_{\varphi}^{[n]}$ on $H^{2}(\beta)$. Then we obtain $C_{\varphi}^{[1]^{*}}$ on the Hardy, Bergman and Dirichlet spaces for a rational self-map $\varphi$ of $\mathbb{U}$. We start with a summary discussion on boundedness.

Corollary 4.1. Suppose that there exists a constant $C>0$ such that for every non-negative integer $m$, the weight sequence of $H^{2}(\beta)$ satisfies $\frac{\beta_{m+1}}{\beta_{m}} \leq C$ and let $\varphi$ be an analytic self-map of $\mathbb{U}$. Then

(i) $Q$ is a Hilbert-Schmidt operator on $H^{2}(\beta)$.

(ii) If $C_{\varphi}$ is bounded on $H^{2}(\beta)$, then $C_{\varphi}^{[n]}$ is Hilbert-Schmidt on $H^{2}(\beta)$.

Proof. (i) Let $\left\{e_{m}\right\}_{m=1}^{\infty}$ defined by $e_{m}(z)=\frac{1}{\beta_{m}} z^{m}$ be the orthonormal basis for $H^{2}(\beta)$. Then $Q e_{m}(z)=\frac{1}{(m+1) \beta_{m}} z^{m+1}$ and we have

$$
\sum_{m=0}^{\infty}\left\|Q e_{m}\right\|_{\beta}^{2}=\sum_{m=0}^{\infty} \frac{1}{(m+1)^{2} \beta_{m}^{2}} \beta_{m+1}^{2} \leq C^{2} \sum_{m=0}^{\infty} \frac{1}{(m+1)^{2}}<\infty .
$$

Therefore $Q$ is a Hilbert-Schmidt operator on $H^{2}(\beta)$. Part (ii) follows immediately from $C_{\varphi}^{[n]}=C_{\varphi} Q^{n}$ and the fact that Hilbert-Schmidt operators form a two-sided $*$-ideal in the $C^{*}$-algebra of bounded operators on $H^{2}(\beta)$.

As a consequence of Littlewood's Subordination Principle, every composition operator on the Hardy space is bounded. By Proposition 3.4 in [11], this fact also holds for every composition operator on the Bergman space. In view of Corollary 4.1, for the generalized composition operators on the aforementioned spaces we have even more.

Corollary 4.2. Let $\varphi$ be an analytic self-map of $\mathbb{U}$. Then $C_{\varphi}^{[n]}$ is a HilbertSchmidt operator on the Hardy and Bergman spaces.

Now we consider the following theorem on the adjoint of the generalized composition operators on the weighted Hardy spaces. 
Theorem 4.3. Let $B$ denote the backward shift operator on $H^{2}(\beta)$ and suppose $Q$ is bounded on $H^{2}(\beta)$. Then

(i) $Q^{*}=B Q B$.

(ii) $C_{\varphi}^{[n]^{*}}=(B Q B)^{n} C_{\varphi}^{*}$.

(iii) For $f \in H^{2}(\beta)$ with $f(z)=\sum_{j=0}^{\infty} a_{j} z^{j}$,

$$
Q^{*} f(z)=\sum_{j=1}^{\infty} \frac{1}{j} \frac{\beta_{j}^{2}}{\beta_{j-1}^{2}} a_{j} z^{j-1}
$$

Proof. $(i)$ Let $j$ and $m$ be arbitrary non-negative integers and define $e_{j}(z)=\frac{1}{\beta_{j}} z^{j}$. Then $\left\{e_{j}\right\}$ is an orthonormal basis for $H^{2}(\beta)$ and

$$
Q e_{j}(z)=\int_{0}^{z} e_{j}(w) d w=\frac{\beta_{j+1}}{(j+1) \beta_{j}} e_{j+1}(z) .
$$

By considering two cases $j=m$ and $j \neq m$ separately, we have

$$
\begin{aligned}
\left\langle Q^{*} U e_{j}, e_{m}\right\rangle_{\beta} & =\left\langle Q^{*} e_{j+1}, e_{m}\right\rangle_{\beta}=\left\langle e_{j+1}, Q e_{m}\right\rangle_{\beta} \\
& =\left\langle e_{j+1}, \frac{\beta_{m+1}}{(m+1) \beta_{m}} e_{m+1}\right\rangle_{\beta}=\left\langle\frac{\beta_{j+1}}{(j+1) \beta_{j}} e_{j+1}, e_{m+1}\right\rangle_{\beta} \\
& =\left\langle Q e_{j}, U e_{m}\right\rangle_{\beta}=\left\langle B Q e_{j}, e_{m}\right\rangle_{\beta},
\end{aligned}
$$

where $U$ is the unilateral shift operator on $H^{2}(\beta)$. Therefore, $Q^{*} U=B Q$ and hence $Q^{*} U B=B Q B$. Moreover, the equality

$$
\left\langle Q^{*} e_{0}, e_{j}\right\rangle_{\beta}=\left\langle e_{0}, Q e_{j}\right\rangle_{\beta}=\left\langle e_{0}, \frac{\beta_{j+1}}{(j+1) \beta_{j}} e_{j+1}\right\rangle_{\beta}=0
$$

implies $Q^{*} e_{0}=0$. Since every constant function is in $\operatorname{ker} B$ and $U B=I$ on the subspace $\left\{f \in H^{2}(\beta): f(0)=0\right\}$ of $H^{2}(\beta)$, it follows that for arbitrary $f \in H^{2}(\beta)$,

$$
\begin{aligned}
B Q B f & =Q^{*} U B(f-f(0))+Q^{*} U B(f(0))=Q^{*}(f-f(0)) \\
& =Q^{*} f-Q^{*}(f(0))=Q^{*} f .
\end{aligned}
$$

(ii) Observe that $C_{\varphi}^{[n]^{*}}=\left(C_{\varphi} Q^{n}\right)^{*}=(B Q B)^{n} C_{\varphi}^{*}$.

(iii) Let $f_{j}(z)=z^{j}$. Then, (4.2) implies that for $j \geq 1$,

$$
\begin{aligned}
Q^{*} f_{j} & =\beta_{j} Q^{*} e_{j}=\beta_{j} B Q B e_{j}=\beta_{j} B Q e_{j-1}=\beta_{j} \frac{\beta_{j}}{j \beta_{j-1}} B e_{j} \\
& =\frac{\beta_{j}^{2}}{j \beta_{j-1}} e_{j-1}=\frac{\beta_{j}^{2}}{j \beta_{j-1}^{2}} f_{j-1},
\end{aligned}
$$

and since $Q^{*} e_{0}=0$, we obtain (4.1).

For any self-map $\varphi$ of $\mathbb{U}$ and $f$ belonging to the weighted Hardy space $H^{2}(\beta)$ we see that,

$$
\left(C_{\varphi}^{[1]^{*}} f\right)(0)=\left\langle C_{\varphi}^{[1]^{*}} f, \frac{1}{\beta_{0}^{2}}\right\rangle_{H^{2}(\beta)}=\left\langle f, \frac{1^{[1]} \circ \varphi}{\beta_{0}^{2}}\right\rangle_{H^{2}(\beta)}=\left\langle f, \frac{\varphi}{\beta_{0}^{2}}\right\rangle_{H^{2}(\beta)}
$$


In the remaining of this section we give an expression for the adjoint of a generalized composition operator $C_{\varphi}^{[1]}$ with rational symbol $\varphi$ on the Hardy, Bergman and Dirichlet spaces.

Theorem 4.4. Let $C_{\varphi}^{[1]}$ be a generalized composition operator with rational symbol on $H^{2}(\mathbb{U})$. Then for each non-zero $z \in \operatorname{reg}\left(\varphi_{e}\right) \cap \mathbb{U}$,

$$
\left(C_{\varphi}^{[1]^{*}} f\right)(z)=f(0) K_{\varphi(0), 1}(z)+\sum_{j=1}^{d} B_{0} C_{\sigma_{j}} Q B_{0} f(z) .
$$

Furthermore, $\left(C_{\varphi}^{[1]^{*}} f\right)(0)=\langle f, \varphi\rangle_{H^{2}}$.

Proof. Let $z \in \operatorname{reg}\left(\varphi_{e}\right) \cap \mathbb{U}$ be non-zero. For $h_{0}(z)=\frac{1}{1-\overline{\varphi(0)} z}$, one can use elementary calculus to observe that

$$
B_{0} Q B_{0} h_{0}(z)=\frac{1}{z} \log \frac{1}{1-\overline{\varphi(0)} z} .
$$

For $h_{j}(z)=z \sigma_{j}^{\prime}(z)\left(B_{0} f\right)\left(\sigma_{j}(z)\right)$, we have

$$
\begin{aligned}
Q B_{0} h_{j}(z) & =\int_{0}^{z} \sigma_{j}^{\prime}(w)\left(B_{0} f\right)\left(\sigma_{j}(w)\right) d w \\
& =\left(Q B_{0} f\right)\left(\sigma_{j}(z)\right)-\left(Q B_{0} f\right)\left(\sigma_{j}(0)\right),
\end{aligned}
$$

which implies

$$
B_{0} Q B_{0} h_{j}(z)=\frac{C_{\sigma_{j}} Q B_{0} f(z)-C_{\sigma_{j}} Q B_{0} f(0)}{z}=B_{0} C_{\sigma_{j}} Q B_{0} f(z) .
$$

Therefore, using (4.3), (4.4), Theorem 2.3 and Theorem 4.3, we have

$$
\begin{aligned}
C_{\varphi}^{[1]^{*}} f(z) & =\left(C_{\varphi} Q\right)^{*} f(z)=Q^{*} C_{\varphi}^{*} f(z)=B_{0} Q B_{0} C_{\varphi}^{*} f(z) \\
& =B_{0} Q B_{0}\left(f(0) h_{0}+\sum_{j=1}^{d} h_{j}\right)(z) \\
& =f(0) \frac{1}{z} \log \frac{1}{1-\overline{\varphi(0)} z}+\sum_{j=1}^{d} B_{0} C_{\sigma_{j}} Q B_{0} f(z) \\
& =f(0) K_{\varphi(0), 1}(z)+\sum_{j=1}^{d} B_{0} C_{\sigma_{j}} Q B_{0} f(z) .
\end{aligned}
$$

Theorem 4.5. Let $C_{\varphi}^{[1]}$ be a generalized composition operator with rational symbol on $A^{2}(\mathbb{U})$. Then for each $z \in \operatorname{reg}\left(\varphi_{e}\right) \cap \mathbb{U}$,

$$
\left(C_{\varphi}^{[1]^{*}} f\right)(z)=f(0) K_{\varphi(0), 1}(z)+\sum_{j=1}^{d} W_{\sigma_{j}^{\prime}, \sigma_{j}} B_{0}^{2} Q f(z) .
$$


Proof. Assume that $T_{0}$ and $T_{1}$ are the generalized composition operator $C_{\varphi}^{[1]}$ on $H^{2}(\mathbb{U})$ and $A^{2}(\mathbb{U})$ respectively, and let $T: A^{2}(\mathbb{U}) \rightarrow H^{2}(\mathbb{U})$ be the corresponding isometric isomorphism defined in Theorem 2.1. Then by (2.2) for $z \neq 0$,

$$
T(T f)(z)=B_{0} Q f(z)=\frac{F(z)}{z},
$$

and since $B_{0} Q f(0)=0$, it follows from Theorem 4.4 that

$$
\begin{aligned}
T_{0}^{*}(T(T f))(z) & =T_{0}^{*}\left(B_{0} Q f\right)(z) \\
& =\frac{1}{z} \log \frac{1}{1-\overline{\varphi(0)} z} f(0)+\sum_{j=1}^{d} B_{0} C_{\sigma_{j}} Q B_{0}^{2} Q f(z) .
\end{aligned}
$$

For every $h \in A^{2}(\mathbb{U})$, we have $\left(z B_{0} h(z)\right)^{\prime}=(h(z)-h(0))^{\prime}=h^{\prime}(z)$. Therefore by (2.1), (4.5) and Corollary 2.2, for every non-zero $z \in \operatorname{reg}\left(\varphi_{e}\right) \cap \mathbb{U}$ we have

$$
\begin{aligned}
\left(T_{1}^{*} f\right)(z) & =T^{-1}\left(T^{-1}\left(T_{0}^{*}(T(T f))\right)\right)(z)=\left(z T_{0}^{*} B_{0} Q f(z)\right)^{\prime} \\
& =f(0)\left(\log \frac{1}{1-\overline{\varphi(0)} z}\right)^{\prime}+\sum_{j=1}^{d}\left(Q B_{0}^{2} Q f\left(\sigma_{j}(z)\right)\right)^{\prime} \\
& =f(0) \frac{\overline{\varphi(0)}}{1-\overline{\varphi(0)} z}+\sum_{j=1}^{d} \sigma_{j}^{\prime}(z) B_{0}^{2} Q f\left(\sigma_{j}(z)\right) \\
& =f(0) \frac{\overline{\varphi(0)}}{1-\overline{\varphi(0)} z}+\sum_{j=1}^{d} W_{\sigma_{j}^{\prime}, \sigma_{j}} B_{0}^{2} Q f(z) \\
& =f(0) K_{\varphi(0), 1}(z)+\sum_{j=1}^{d} W_{\sigma_{j}^{\prime}, \sigma_{j}} B_{0}^{2} Q f(z) .
\end{aligned}
$$

The statement of the theorem also holds for $z=0$ by analyticity.

Now let $\varphi(z)=\frac{a z+b}{c z+d}$ be a linear fractional self-map of $\mathbb{U}$ and define the associated linear fractional transformation by $\sigma(z)=\frac{\bar{a} z-\bar{c}}{-\bar{b} z+\bar{d}}$, which is known as the Krein adjoint of $\varphi$. It was shown by Cowen [4] that $\varphi$ is a self-map of $\mathbb{U}$ if and only if $\sigma$ is a self-map of $\mathbb{U}$. Now it can be seen from Theorem 4.5 that the adjoint of a generalized composition operator with linear fractional symbol has an explicit formula on the Bergman space as follows.

Corollary 4.6. Let $C_{\varphi}^{[1]}$ be a generalized composition operators on $A^{2}(\mathbb{U})$ with linear fractional symbol $\varphi(z)=\frac{a z+b}{c z+d}$. Then for each $f \in A^{2}(\mathbb{U})$,

$$
\left(C_{\varphi}^{[1]^{*}} f\right)(z)=-f(0) \frac{\bar{a}}{\bar{a} z-\bar{c}}+\frac{\bar{a} \bar{d}-\bar{b} \bar{c}}{(\bar{a} z-\bar{c})^{2}} F\left(\frac{\bar{a} z-\bar{c}}{-\bar{b} z+\bar{d}}\right)
$$

where $F$ is the anti-derivative of $f$ with $F(0)=0$. Moreover, $\left(C_{\varphi}^{[1]^{*}} f\right)(0)=$ $\langle f, \varphi\rangle_{A^{2}}$. 
Theorem 4.7. Let $\mathbb{U} \subset \operatorname{Reg}\left(\varphi_{e}\right)$ and suppose that $C_{\varphi}^{[1]}$ is a generalized composition operator on $\mathcal{D}$ with rational symbol. Then $C_{\varphi}^{[1]}$ is Hilbert-Schmidt on $\mathcal{D}$ and for $f \in \mathcal{D}$ and $z \neq 0$,

$$
\left(C_{\varphi}^{[1]^{*}} f\right)(z)=f(0) K_{\varphi(0), 1}(z)+\sum_{j=1}^{d} \sigma_{j}^{\prime}(0) f^{\prime}\left(\sigma_{j}(0)\right)+\sum_{j=1}^{d} Q B_{0}^{2} C_{\sigma_{j}} f(z) .
$$

Proof. Any self-map of $\mathbb{U}$ with bounded multiplicity induces a bounded composition operator on the Dirichlet space (see [10, Proposition 1.1]). Therefore $C_{\varphi}$ is bounded on $\mathcal{D}$. On the other hand, by Corollary $4.1(i), Q$ is a Hilbert-Schmidt operator on $\mathcal{D}$. Consequently, $C_{\varphi}^{[1]}=C_{\varphi} Q$ is a Hilbert-Schmidt operator on $\mathcal{D}$.

For $f$ in the Dirichlet space with Maclaurin series expansion $f(z)=\sum_{n=0}^{\infty} a_{n} z^{n}$, by using (4.1) we have

$$
\begin{aligned}
Q^{*} f(z) & =\sum_{n=1}^{\infty} \frac{1}{n} \frac{\beta_{n}^{2}}{\beta_{n-1}^{2}} a_{n} z^{n-1}=a_{1}+\sum_{n=2}^{\infty} \frac{1}{n-1} a_{n} z^{n-1} \\
& =a_{1}+\int_{0}^{z} \sum_{n=2}^{\infty} a_{n} t^{n-2} d t=a_{1}+\int_{0}^{z} B_{0}^{2} f(t) d t \\
& =f^{\prime}(0)+Q B_{0}^{2} f(z) .
\end{aligned}
$$

Moreover,

$$
\begin{aligned}
Q^{*} K_{\varphi(0)}(z) & =\left\langle Q^{*} K_{\varphi(0)}, K_{z}\right\rangle=\left\langle K_{\varphi(0)}, Q K_{z}\right\rangle \\
& =\overline{\left\langle Q K_{z}, K_{\varphi(0)}\right\rangle}=\overline{Q K_{z}(\varphi(0))} \\
& =\overline{2 \overline{\varphi(0)}}+\frac{1-\overline{\varphi(0)} z}{z} \log (1-\overline{\varphi(0)} z)=K_{\varphi(0), 1}(z) .
\end{aligned}
$$

From equality (4.6), Theorem 2.4 and the fact that the constant functions are in $\operatorname{ker} Q^{*}$, we conclude that

$$
\left(C_{\varphi}^{[1]^{*}} f\right)(z)=Q^{*}\left(C_{\varphi}^{*} f\right)(z)=f(0) K_{\varphi(0), 1}(z)+\sum_{j=1}^{d} \sigma_{j}^{\prime}(0) f^{\prime}\left(\sigma_{j}(0)\right)+\sum_{j=1}^{d} Q B_{0}^{2} C_{\sigma_{j}} f(z)
$$

Acknowledgement. The authors wish to express their gratitude to the anonymous referee for giving valuable comments and suggestions, which improved the paper.

\section{REFERENCES}

1. P.S. Bourdon and J.H. Shapiro, Adjoints of rationally induced composition operators, J. Funct. Anal. 255 (2008), no. 8, 1995-2012.

2. C.C. Cowen, Linear fractional composition operators on $H^{2}$, Integral Equations Operator Theory 11 (1988), no. 2, 151-160.

3. C.C. Cowen and E.A. Gallardo-Gutiérrez, A new class of operators and a description of adjoints of composition operators, J. Funct. Anal. 238 (2006), no. 2, 447-462.

4. C.C. Cowen and B.D. MacCluer, Composition Operators on Spaces of Analytic Functions, CRC Press, Boca Raton, 1995. 
5. P.L. Duren, Theory of $H^{p}$ Spaces, Academic Press, New York, 1970.

6. E. Gallardo-Gutiérrez and A. Montes-Rodríguez, Adjoints of linear fractional composition operators on the Dirichlet space, Math. Ann. 327 (2003), no. 1, 117-134.

7. A. Goshabulaghi and H. Vaezi, Adjoints of rationally induced composition operators on Bergman and Dirichlet spaces, Turkish J. Math. 38 (2014), no. 5, 862-871.

8. C. Hammond, J. Moorhouse and M.E. Robbins, Adjoints of composition operators with rational symbol, J. Math. Anal. Appl. 341 (2008), no. 1, 626-639.

9. P.R. Hurst, Relating composition operators on different weighted Hardy spaces, Arch. Math. (Basel) 68 (1997), no. 6, 503-513.

10. M. Jovović and B.D . MacCluer, Composition operators on Dirichlet spaces, Acta Sci. Math. (Szeged) 63 (1997), no. 1-2, 229-247.

11. B.D. MacCluer and J.H. Shapiro, Angular derivatives and compact composition operators on the Hardy and Bergman spaces, Canad. J. Math. 38 (1986), 878-906.

12. M.J. Martín and D. Vukotić, Adjoints of composition operators on Hilbert spaces of analytic functions, J. Funct. Anal. 238 (2006), no. 1, 298-312.

13. J.N. McDonald, Adjoints of a class of composition operators, Proc. Amer. Math. Soc. 131 (2003), no. 2, 601-606.

14. J.H. Shapiro, Composition Operators and Classical Function Theory, Springer-Verlag, Berlin, 1993.

15. A.K. Sharma, Generalized composition operators between Hardy and weighted Bergman spaces, Acta Sci. Math. (Szeged), 78 (2012), no. 1-2, 187-211.

16. X. Zhu, Products of differentiation, composition and multiplication operator from Bergman type spaces to Bers spaces, Integral Transforms Spec. Funct. 18 (2007), no. 3-4, 223-231.

Department of Mathematics, Faculty of Mathematical Sciences, University of Tabriz, P. O. Box: 51666, Tabriz, Iran.

E-mail address: a_goshabulaghi@tabrizu.ac.ir

E-mail address: hvaezi@tabrizu.ac.ir 